in

NASA W 6067

\title{
ASYMPTOTIC FORM FOR THE
} CROSS SECTION FOR THE COULOMB INTERACTING REARRANGEMENT COLLISIONS

K. OMIDVAR

(NASA-TM-X-66184) : ASY EPTOTIC FORM FOR THE CROSS SECTION FOR THE COULOHB INTERACTING REARRANGEHENT COLLISIONS (NASA) 10 - $\mathrm{HC}$ CSCL $20 \mathrm{H}$ $\lambda$

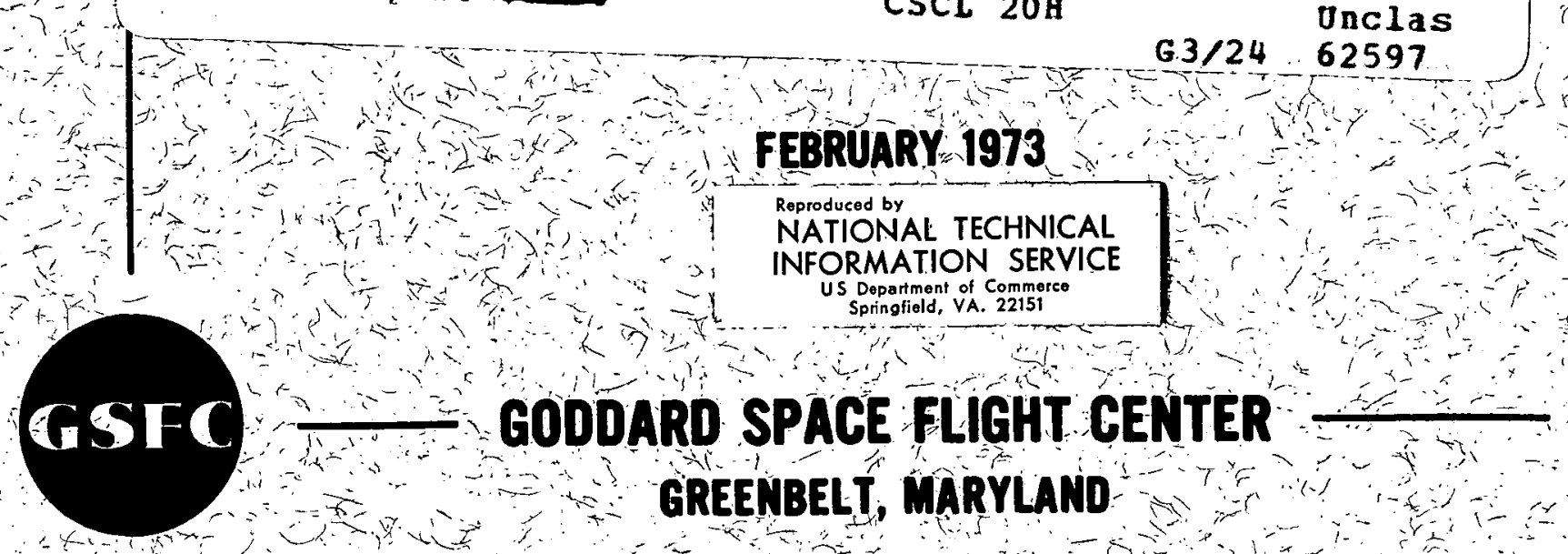

\section{FEBRUARY 1973}

NATIONAL TECHNICAL

NFORMATION SERVICE

Springfield, VA. 22151 


\section{ASYMPTOTIC FORM FOR THE CROSS SECTION \\ FOR THE COULOMB INTERACTING \\ REARRANGEMENT COLLISTONS}

K. Omidvar

Theoretical Studies Branch, NASA/Goddard Space

Flight Center, Greenbelt, Maryland 20771

It is shown that in a rearrangement collision leading to the formation of the highly excited hydrogenlike states the cross section in all orders of the Born approximation behaves as $1 / \mathbf{n}^{2}$, with $n$ the principal quantum number, thus invalidating the BrinkmanKramers approximation for large n. Similarly, in high energy inelastic electron-hydrogenlike atom collisions the exchange cross section for sufficiently large $n$ dominates the direct excitation cross section.

We consider the collision of two like and one unlike charged particles 1,2 , 3 with masses $m_{1}, m_{2}, m_{3}$ and charges $z_{1} e, z_{2} e, z_{3} e$, respectively, where $e$ is the absolute value of the electronic charge. The collision is represented by $1+(2+3) \rightarrow(1+3)+2$ where $(2+3)$ and $(1+3)$ represent the hydrogenlike 
states of 2 and 3 , and 1 and 3 , respectively. We assume that $(2+3)$ is in the ground state, but $(1+3)$ is in an arbitrary state including the continuum. Examples would be capture of an electron by a proton incident on atomic hydrogen, and the exchange effect in scattering of electrons by atomic hydrogen.

The collision amplitude in the $\mathrm{M}^{\mathrm{th}}$ order of the Born approximation is given by ${ }^{1}$

$$
\left.T_{f}{ }^{M+1}\right)=\left\langle\exp \left(i k_{2} \cdot \mathbf{r}_{2}\right) \Psi\left(f, \mathbf{r}_{13}\right)\left|V_{f}\left(G_{o} V_{i}\right)^{M}\right| \exp \left(i k_{1} \cdot \mathbf{r}_{1}\right) \Psi\left(i, \mathbf{r}_{23}\right)\right\rangle
$$

where the subscript $f$ on the left hand side designates that the post interaction form has been used for the amplitude. $\Psi\left(\mathbf{i}, \mathbf{r}_{23}\right)$ and $\Psi\left(f, \mathbf{r}_{13}\right)$ are the bound states of $(2+3)$ and $(1+3)$ where $\mathbf{r}_{23}$ and $\mathbf{r}_{13}$ are vectors connecting particles 2 and 1 respectively to particle 3 . The vectors $\mathbf{r}_{1}$ and $\mathbf{r}_{2}$ connect the centers of masses of $(2+3)$ and $(1+3)$ to the particles 1 and 2 , and vectors $k_{1}$ and $k_{2}$ are the propagation vectors of particles 1 and 2 with respect to the centers of masses of $(2+3)$ and $(1+3)$, respectively. $\left|\mathbf{k}_{2}\right|$ is related to $\left|\mathbf{k}_{1}\right|$ through

$$
\frac{\hbar^{2} k_{2}^{2}}{2 \mu_{2}}=\frac{\hbar^{2} k_{1}^{2}}{2 \mu_{1}}+E(2,3)-E(1,3), \mu_{i}=\frac{m_{i}\left(m_{j}+m_{k}\right)}{m_{i}+m_{j}+m_{k}}
$$

where $E(2,3)$ and $E(1,3)$ are the energies of $(2+3)$ and $(1+3)$ states. Finally, $V_{f}=V_{12}+V_{23}$, and $V_{i}=V_{12}+V_{13}$, where $V_{i j}$ is the potential between $i$ and 
$j$ particles, and $G_{0}$ is the three body Green's function for outgoing waves. It should be noted that $\mathrm{V}_{12}$ is repulsive, while $\mathrm{V}_{13}$ and $\mathrm{V}_{23}$ are attractive potentials. The rearrangement cross section is related to the rearrangement amplitude through the relationship

$$
\sigma=\frac{\mu_{1} \mu_{2}}{2 \pi \hbar^{4}}\left(\frac{\mathrm{k}_{2}}{\mathrm{k}_{1}}\right) \int|\mathrm{T}|^{2} \mathrm{~d}\left(\hat{\mathrm{k}}_{1} \cdot \hat{\mathrm{k}}_{2}\right)
$$

We first consider the first Born approximation which corresponds to $M=0$ in (1). The cross section when only $\mathrm{V}_{23}$ is taken into account, commonly called the Brinkman-Kramers cross section, was originally calculated by Brinkman and Kramers ${ }^{2}$ taking the ground state as the final state. Calculations for the excited states as the final states have been carried out by May ${ }^{3}$ and Omidvar ${ }^{4}$. These calculations indicate that at high relative incident energies the cross section behaves as $\mathrm{n}^{-3}$ with $\mathrm{n}$ the principal quantum number of the final excited state. This behavior has also been predicted by Oppenheimer ${ }^{5}$.

We shall consider here the part of the amplitude coming from the $V_{12}$ potential that can be written ${ }^{6}$

$$
\begin{aligned}
& \mathrm{T}_{\mathrm{f}}^{(1)}\left(\mathrm{V}_{12}\right)=4 \pi \mathrm{Z}_{1} \mathrm{Z}_{2} \mathrm{e}^{2} \int \mathrm{U}^{*}(\mathrm{f}, \mathrm{C}-\mathbf{p}) \mathrm{U}(\mathrm{i}, \mathbf{B}-\mathbf{p}) \frac{\mathrm{d} \mathbf{p}}{\mathrm{p}^{2}}, \\
& \mathrm{C}=\mathbf{k}_{1}-\frac{\mu_{13}}{\mathrm{~m}_{3}} \mathbf{k}_{2}, \mathbf{B}=\frac{\mu_{23}}{\mathrm{~m}_{3}} \mathbf{k}_{1}-\mathbf{k}_{2}, \mu_{\mathrm{ij}}=\frac{\mathrm{m}_{\mathrm{i}} \mathrm{m}_{\mathrm{j}}}{\mathrm{m}_{\mathrm{i}}+\mathrm{m}_{\mathrm{j}}}
\end{aligned}
$$


where

$$
\mathrm{U}(\mathbf{j}, \mathbf{q})=(2 \pi)^{-3 / 2} \int \exp (\mathrm{i} \mathbf{q} \cdot \mathbf{r}) \Psi(\mathbf{j}, \mathbf{r}) \mathrm{d} \mathbf{r}
$$

In this article this amplitude is evaluated for large $\mathrm{n}$ and it is shown that the asymptotic form of the cross section with respect to $\mathrm{n}$ is dominated by this amplitude.

When the bound states are expressed in parabolic coordinates we have ${ }^{4}$

$$
\begin{aligned}
& \mathrm{U}\left(\mathrm{nn}{ }_{1} \mathrm{~m}, \mathbf{q}\right)=\frac{\delta(\mathrm{m}, \mathrm{o}) \sqrt{\mathrm{n}}}{\pi} \frac{(\alpha / 2)^{5 / 2}}{|\omega|^{4}}\left(\frac{\omega^{*}}{\omega}\right)^{2 \mathrm{n}_{1}}, \\
& \alpha=\mu_{\mathrm{ij}} Z_{\mathrm{i}} Z_{j} /\left(\mathrm{m}_{\mathrm{e}} \mathrm{na_{o }}\right), \omega=\frac{1}{2}(\alpha-\mathrm{iq}), \hat{\mathrm{z}}=\hat{\mathrm{q}},
\end{aligned}
$$

with $n_{1}$ and $m$ the Stark and the absolute value of the magnetic quantum numbers, $\mathrm{m}_{\mathrm{e}}$ the electronic mass, and $\mathrm{a}_{\mathrm{o}}$ the Bohr radius. In (6) the spacial quantization axis is taken along $\mathbf{q}$.

Taking the ground state as the initial state and designating the final state by $\mathrm{n} \mathrm{n}_{1} \mathrm{~m}$, through (6) Eq. (4) can be written

$$
\begin{aligned}
\mathrm{T}_{\mathrm{nn}_{1} \mathrm{~m}}(1)\left(\mathrm{V}_{12}\right) & =\delta(\mathrm{m}, 0) 32 \pi^{-1} \mathrm{Z}_{1} \mathrm{Z}_{2} \mathrm{e}^{2}\left(a_{0} \alpha\right)^{5 / 2} \sqrt{\mathrm{n}} \mathrm{g} \\
a_{0} & =\mu_{23} \mathrm{Z}_{2} \mathrm{Z}_{3} /\left(\mathrm{m}_{\mathrm{e}} \mathrm{a}_{0}\right), a=\mu_{13} \mathrm{Z}_{1} \mathrm{Z}_{3} /\left(\mathrm{m}_{\mathrm{e}} \mathrm{n} \mathrm{a}_{0}\right)
\end{aligned}
$$




$$
I=\int \frac{d \mathbf{p}}{\mathbf{p}^{2}\left[\alpha_{0}^{2}+(\mathbf{B}-\mathbf{p})^{2}\right]^{2}\left[\alpha^{2}+(\mathbf{C}-\mathbf{p})^{2}\right]^{2}}\left(\frac{\alpha-i|\mathbf{C}-\mathbf{p}|}{\alpha+\mathbf{i}|\mathbf{C}-\mathbf{p}|}\right)^{2 \mathrm{n}_{1}}
$$

For $\mathrm{n}_{1}=0$ and $\mathrm{n} \rightarrow \infty$ the integration in (8) can be affected by a delta function integration. This leads to

$$
\mathrm{T}_{\text {nom }}(1)\left(\mathrm{V}_{12}\right) \approx \frac{\delta(\mathrm{m}, 0) 32 \pi \mathrm{Z}_{1} \mathrm{Z}_{2} \mathrm{e}^{2} \sqrt{\mathrm{n}} \alpha_{0}^{5 / 2} \alpha^{3 / 2}}{\mathrm{C}^{2}\left[a_{0}{ }^{2}+(B-C)^{2}\right]^{2}}, \alpha \rightarrow 0
$$

For $n_{1} \neq 0$ this method of integration is not applicable.

For evaluation of $\mathfrak{g}$ for arbitrary $\mathbf{n}_{1}$ we introduce in Eq. (8) $\mathbf{q}=\mathbf{C}-\mathbf{p}$ and make use of the Feynman's parametric integration method ${ }^{7}$. Then ${ }^{9}$ can be written

$$
\begin{gathered}
g=-\frac{\partial g_{1}}{\partial\left(a_{0}^{2}\right)}, g_{1}=4 \pi \int_{0}^{1} \mathrm{dx}\left(g_{2}+\mathrm{i} g_{3}\right), \\
g_{2}=\frac{1}{2} \int_{-\infty}^{+\infty} \frac{(\alpha-\mathrm{iq})^{2\left(\mathrm{n}_{1}-1\right)}}{(\alpha+\mathrm{iq})^{2\left(\mathrm{n}_{1}+1\right)}} \frac{\mathrm{q}^{2} \mathrm{~d} \mathrm{q}}{\left(\mathrm{q}^{2}+\Delta\right)^{2}-4 \mathrm{Q}^{2} \mathrm{q}^{2}}, \\
g_{3}=-\int_{0}^{\infty} \frac{\mathrm{sin}\left(4 \mathrm{n}_{1} \phi\right)}{\left(\alpha^{2}+\mathrm{q}^{2}\right)^{2}} \frac{\mathrm{q}^{2} \mathrm{dq}}{\left(\mathrm{q}^{2}+\Delta\right)^{2}-4 \mathrm{Q}^{2} \mathrm{q}^{2}}, \phi=\mathrm{tan}^{-1} \mathrm{q} / \alpha \\
g_{3}=-\sum_{\nu=0}^{2 \mathrm{n}_{1}-1}\left(\frac{4 \mathrm{n}_{1}}{2 \nu+1}\right)_{(-)^{\nu} \alpha^{4 \mathrm{n}_{1}-1-2 \nu}} \int_{0}^{\infty} \frac{\mathrm{q}^{2 \nu+3} \mathrm{dq}}{\left.\left(\alpha^{2}+\mathrm{q}^{2}\right)^{2\left(\mathrm{n}_{1}+1\right)}\left[\mathrm{q}^{2}+\Delta\right)^{2}-4 \mathrm{Q}^{2} \mathrm{q}^{2}\right]},
\end{gathered}
$$




$$
\Delta=\left[a_{0}^{2}+(\mathbf{B}-\mathbf{C})^{2}\right] \mathbf{x}+\mathbf{C}^{2}(1-\mathbf{x}), \mathbf{Q}=-\mathbf{B} \mathbf{x}+\mathbf{C}
$$

$g_{2}$ can be evaluated by a contour integration. The evaluation of $\mathfrak{I}_{2}$ for $\mathbf{n}_{1}=0$ and $a-0$ gives $9_{2} \approx-\pi /\left(4 a \triangle^{2}\right)$. Substitution of this result in (10) leads to a result identical to (9). For $n_{1} \neq 0$ and $\alpha \rightarrow 0,9_{2}$ does not show any pole with respect to $a$, and remain finite as $\mathbf{n} \rightarrow \infty$.

To evaluate $9_{3}$ we use the form (12b). By an ordinary integration we find that

$$
g_{3} \approx \frac{c_{n_{1}}}{2 a \Delta^{2}}, c_{n_{1}}=\sum_{\nu=0}^{2 n_{1}-1}\left(\begin{array}{c}
4 n_{1} \\
2 \nu+1
\end{array}\right) \sum_{\lambda=0}^{\nu_{+} 1} \frac{(-)^{\lambda}}{2 n_{1}+1-\lambda}\left(\begin{array}{c}
\nu+1 \\
\lambda
\end{array}\right), \alpha \rightarrow 0
$$

Substitution of this result in (10) gives

$$
T_{n n_{1} m}\left(V_{12}\right)=\frac{\delta(m, 0) 32 \pi Z_{1} Z_{2} e^{2} v_{n} a_{0}^{5 / 2} a^{3 / 2}}{C^{2}\left[a_{0}^{2}+(B-C)^{2}\right]^{2}} \frac{2 \mathrm{i} c_{n_{1}}}{\pi}, n_{1}=1,2,3, \cdots, a \rightarrow 0
$$

By substituting from (9) and (15) into (3), neglecting contribution due to the $\mathrm{V}_{23}$ potential, and summing the right hand side of (3) with respect to $\mathrm{n}_{1}$ and $\mathrm{m}$, we find an expression for the total cross section for capture into an excited state n. This is given by

$$
\begin{aligned}
\frac{\sigma(\mathrm{n})}{\pi \mathrm{a}_{0}{ }^{2}} & \approx 2^{9}\left(\frac{\mu_{1} \mu_{2}}{\mathrm{~m}_{\mathrm{e}}{ }^{2}}\right)\left(\mathrm{Z}_{1} \mathrm{Z}_{2}\right)^{2} \alpha_{0}{ }^{5} \mathrm{n} \alpha^{3}\left[1+\frac{4}{\pi^{2}} \sum_{\mathrm{n}_{1}=1}^{\mathrm{n}-1} \mathrm{c}_{\mathrm{n}_{1}}^{2}\right] \\
& \times \frac{\mathrm{k}_{2}}{\mathrm{k}_{1}} \int \frac{\mathrm{d}\left(\hat{\mathrm{k}}_{1} \cdot \hat{\mathrm{k}}_{2}\right)}{\mathrm{C}^{4}\left[\alpha_{0}{ }^{2}+(B-C)^{2}\right]^{4}}, \mathrm{n} \rightarrow \infty
\end{aligned}
$$


As $n_{1} \rightarrow \infty, c_{n_{1}}$ approaches zero. This can be shown if $g_{3}$ is expressed through (12a) in its asymptotic form with respect to $n_{1}$ :

$$
g_{3} \approx-\frac{\pi}{\alpha} \int_{0}^{\pi / 2} \frac{\mathrm{q}^{2} \phi \delta(\phi) \mathrm{d} \phi}{\left(\alpha^{2}+\mathrm{q}^{2}\right)\left[\left(\mathrm{q}^{2}+\Delta\right)^{2}-4 \mathrm{Q}^{2} \mathrm{q}^{2}\right]}, \mathrm{n}_{1} \rightarrow \infty
$$

Comparison of (14) and (17) shows that $c_{n_{1}} \rightarrow 0$ as $n_{1} \rightarrow \infty$. Using the explicit form of $c_{n_{1}}$ as given by (14) it is found that

$$
\sum_{n_{1}=1}^{\infty} c_{n_{1}}^{2} \approx 0.616
$$

(Cf. Eq. (16)).

The integrals $\mathfrak{I}_{2}$ and $g_{3}$ can also be evaluated analytically for arbitrary $n$, a providing method hor evaluation of 97 the capture cross sections for the arbitrary excited states. We thus conclude and high impact energies from (16) that due to the repulsive potential the cross section at large $n$ behaves as $1 / \mathrm{n}^{2}$. This has two implications: (1) The cross section according to the Brinkman-Kramers approximation, commonly assumed valid for high principal quantum numbers, is not valid. For the low lying levels this approximation gives too large a cross section. In the case of the symmetric charge exchange between protons and atomic hydrogens Jackson and Schiff ${ }^{6}$ have shown that inclusion of the $\mathrm{V}_{12}$ potential reduces the BK cross sectional values in the energy range of interest by almost an order of magnitude, bringing them closer to the experimental results. Their assumption concerning the $\mathrm{n}$ dependence of the cross section for the excited state is not however correct, and their calculated cross 
sections should be renormalized before they can be compared with the measurements. Similarly, the assumption made by Bates and Dalgarno ${ }^{8}$ that the ratio $\sigma(\mathrm{n} \ell) / \sigma(1 \mathrm{~s})$ is the same for both the Born and the BK approximations is incorrect. $n \ell$ and 1 s designate here the final excited or the ground states. (2) In high energy inelastic scattering of electrons by hydrogenlike atoms the exchange cross section behaving as $1 / \mathrm{n}^{2}$ dominates the direct cross section which behaves as $1 / n^{3}$, a result of significance in plasma and astrophysical calculations.

Considering the second Born approximation, from (1) we find that

$$
\begin{aligned}
& \mathrm{T}_{\mathrm{nn}_{1} \mathrm{~m}}^{(2)}=\frac{2 \mathrm{e}^{4}}{\pi} \iint \frac{\mathrm{dqd} \mathbf{q}^{\prime}\left[Z_{2} Z_{3} \mathrm{U}^{*}\left(\mathrm{nn}_{1} \mathrm{~m}, \mathbf{A}\right)+Z_{1} Z_{2} \mathrm{U}^{*}\left(\mathrm{nn}_{1} \mathrm{~m}, \mathbf{D}\right)\right]\left[Z_{1} Z_{3} \mathrm{U}(100, \mathbf{E})+Z_{1} Z_{2} \mathrm{U}(100, \mathbf{F})\right]}{\left[\frac{\hbar^{2} \mathbf{k}_{2}^{2}}{2 \mu_{2}}+\mathrm{E}(1,3)-\frac{\hbar^{2} \mathrm{q}^{2}}{2 \mu_{2}}-\frac{\hbar^{2} \mathrm{q}^{\prime}{ }^{2}}{2 \mu_{13}}\right]\left(\mathbf{k}_{2}-\mathbf{q}\right)^{2}\left(\mathbf{k}_{1}+\frac{\mu_{13}}{\mathrm{~m}_{3}} \mathbf{q}+\mathbf{q}^{\prime}\right)^{2}} \\
& \mathbf{A}=-\mathbf{q}^{\prime}+\frac{\mu_{13}}{\mathrm{~m}_{3}}\left(\mathbf{k}_{2}-\mathbf{q}\right), \mathbf{D}=-\mathbf{q}^{\prime}-\frac{\mu_{13}}{\mathrm{~m}_{1}}\left(\mathbf{k}_{2}-\mathbf{q}\right) \\
& \mathbf{E}=\frac{\mu_{23}}{\mathrm{~m}_{3}} \mathbf{k}_{1}+\mathbf{q}, \mathbf{F}=-\frac{\mu_{23}}{\mathrm{~m}_{2}} \mathbf{k}_{1}+\frac{\mu_{13}}{\mathrm{~m}_{1}} \mathbf{q}-\mathbf{q}^{\prime}
\end{aligned}
$$

where 100 and $n n_{1} m$ designate the initial and final states. Comparison of (18)

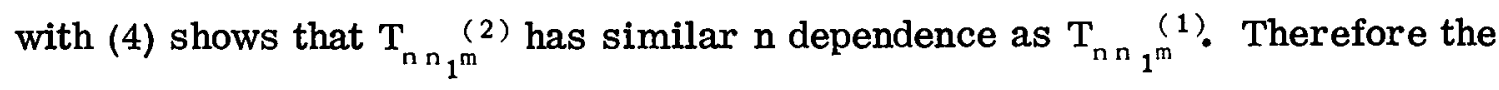
cross section due to the second Born approximation at high $\mathrm{n}$ behaves similarly as $1 / \mathrm{n}^{2}$.

Regarding the higher orders in the Born series it is seen from (1) that the dependence of these orders on the final state is through the first squared bracket in the numerator of the integrand in (18). Then, provided the higher 
order amplitudes have well defined values, their dependence on $\mathrm{n}$ for large $\mathrm{n}$ is the same as for the second order amplitude.

I am indebted to Dr. A. Temkin and Dr. T. G. Northrop for their critical and constructive comments.

\section{REFERENCES}

1. E. Gerjuoy, Phil. Trans. Roy. Soc. (London), 270, 197 (1971).

2. H. C. Brinkmann and H. A. Kramers, Proc. Acad. Sci. Amsterdam 33, $973(1930)$.

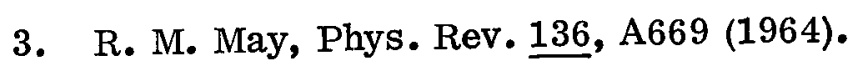

4. K. Omidvar, Phys. Rev. 153, 121 (1966).

5. J. R. Oppenheimer, Phys. Rev. $\underline{31}, 349$ (1928).

6. J. D. Jackson and H. Schiff, Phys. Rev. $\underline{89}, 359$ (1952).

7. R. P. Feynman, Phys. Rev. $\underline{76}, 769$ (1949).

8. D. R. Bates and A. Dalgarno, Proc. Phys. Soc. (London) A66, 972 (1953). 